\title{
Multi-Center Grand Ensemble using Three Operational Ensemble Forecasts
}

\author{
Mio Matsueda ${ }^{1}$, Masayuki Kyouda ${ }^{2}$, H. L. Tanaka ${ }^{3}$ and Tadashi Tsuyuki ${ }^{2}$ \\ ${ }^{1}$ Graduate School of Life and Environmental Sciences, University of Tsukuba, Japan \\ ${ }^{2}$ Numerical Prediction Division, Japan Meteorological Agency, Japan \\ ${ }^{3}$ Center for Computational Sciences, University of Tsukuba, Japan and FRCGC/JAMSTEC, Japan
}

\begin{abstract}
In this study, we investigate the impact of MultiCenter Grand Ensemble (MCGE) forecasts, consisting of three operational ensemble forecasts by the Japan Meteorological Agency (JMA), the National Centers for Environmental Prediction, and the Canadian Meteorological Center. We verified the skill of MCGE forecasts in comparison with that of JMA ensemble forecast using root mean square error, anomaly correlation, and Brier skill score for $500 \mathrm{hPa}$ geopotential height and $850 \mathrm{hPa}$ temperature in the Northern Hemisphere $\left(20^{\circ} \mathrm{N}-90^{\circ} \mathrm{N}\right)$ in September 2005.

Our results show that MCGE forecasts are more skillful than single-center ensemble forecast without considering weight among ensemble members and bias corrections. This implies that considering weight or bias corrections may result in further improvement of MCGE forecasts, specifically in probabilistic forecasts.
\end{abstract}

\section{Introduction}

Ensemble forecast is a collection of a number of forecasts. In the ensemble forecasts, several model forecasts are performed by introducing perturbations in the initial conditions or in the models themselves. Ensemble forecast is employed to achieve two main goals: the first one is to provide that an ensemble average forecast beyond the first few days is more accurate than individual forecast, because the components of the forecast that are most uncertain tend to be averaged out. The second and more important goal is to provide forecasters with an estimation of the reliability of the forecast, which, because of changes in atmospheric predictability, varies from day to day and from region to region (Kalnay 2003).

Operational ensemble forecasts for medium-range forecasting have been performed at several numerical weather prediction (NWP) centers, such as the European Center for Medium-Range Weather Forecasts (ECMWF; Molteni et al. 1996), the National Centers for Environmental Prediction (NCEP; Toth and Kalnay 1997), the Japan Meteorological Agency (JMA; JMA 2002), and the Canadian Meteorological Center (CMC; Houtekamer et al. 1996).

Recently, various types of multiple ensemble forecasts, that are, a multi-model superensemble, poor man's ensemble, a multi-analysis ensemble, a multi-model multi-analysis ensemble and so on, have been brought to the international attention for the purpose of obtaining further useful information from ensemble forecasts (e.g., Krishnamurti et al. 1999; Ziehmann 2001; Buizza et al. 2003; Richardson 2001; Mylne et al. 2003). For

Corresponding author: H. L. Tanaka, Center for Computational Sciences, University of Tsukuba, Tsukuba, 305-8572, Japan. E-mail: tanaka@sakura.cc.tsukuba.ac.jp. (C2006, the Meteorological Society of Japan. example, the aim of a multi-model superensemble is to construct a deterministic forecast, which is more skillful than each NWP center's forecast, by collecting some NWP center's single or several forecasts and then using a multiple regression technique. Also poor man's ensemble regards collections of some NWP center's single or several forecasts as 'one' ensemble forecast. Although we have found that there are many advantages in multi-model or poor man's ensemble, the number of multi-model or poor man's ensemble members is comparable with that of NWP centers by various limitations. If we are able to increase the number of ensemble members further by addition of NWP center's forecasts or using ensemble forecasts, more skillful multiple ensemble may be obtained, especially in probabilistic forecasts.

On the other hand, recent rapid progress of communication networks and information sciences enable us to get vast operational ensemble forecast data from some NWP centers. We hereby can further increase the number of ensemble members as compared with multimodel or poor man's ensemble although there is a limitation of the number of NWP centers which perform operational ensemble forecasts.

The purpose of this study is to investigate the impact of Multi-Center Grand Ensemble (MCGE) technique. In our study, three operational ensemble data from JMA, NCEP, and CMC are collected to construct MCGE forecasts. North American Ensemble Forecast System (NAEFS: see Web site at http://www.emc.ncep. noaa.gov/gmb/ens/NAEFS.html) project has the same design, but the project is now collecting two operational data from NCEP and CMC. THe Observing system Research and Predictability EXperiment (THORPEX: see Web site at http://www.wmo.int/thorpex/) project also has the same design, but the project is now building up the facility of TIGGE (THORPEX Interactive Grand Global Ensemble).

In this paper, we show the verification results of MCGE forecasts in comparison with that of JMA ensemble forecast using Root Mean Square Error (RMSE), Anomaly Correlation (AC), and Brier Skill Score (BSS) for $500 \mathrm{hPa}$ geopotential height (hereafter Z500) and $850 \mathrm{hPa}$ temperature (hereafter T850) in the Northern Hemisphere $\left(20^{\circ} \mathrm{N}-90^{\circ} \mathrm{N}\right)$ in September 2005.

\section{Configurations of operational ensemble forecasts and MCGE forecasts}

In this study, ensemble forecast data from three operational centers, JMA, NCEP, and CMC, are used. The details are summarized in Table 1 . The grid spacing of output data from each center is different. CMC ensemble grid is $1.0^{\circ} \times 1.0^{\circ}$ only and JMA grid is $2.5^{\circ} \times 2.5^{\circ}$ only, although NCEP ensemble grids are $1.0^{\circ} \times 1.0^{\circ}$ and $2.5^{\circ} \times$ $2.5^{\circ}$. So data are interpolated into 2.5 degree grid spacing as a common grid before the verification of MCGE forecasts. 
Table 1. Three ensemble configurations at JMA, NCEP, and CMC.

\begin{tabular}{cccc}
\hline & JMA & NCEP & CMC \\
\hline Model Resol. & T106L40 & T126L28 & TL149L23-41 \\
Init. Perturb. & BVs & BVs & EnKF \\
Init. UTC & 12 & $00,06,12,18$ & 00 \\
Mem./run & 25 & 11 & 17 \\
Mem./day & 25 & 44 & 17 \\
Grid (output) & $2.5^{\circ} \times 2.5^{\circ}$ & $2.5^{\circ} \times 2.5^{\circ}$ & $1.0^{\circ} \times 1.0^{\circ}$ \\
& & $1.0^{\circ} \times 1.0^{\circ}$ & \\
Fore. Leng. (interval) & $216 \mathrm{hr}(12 \mathrm{hr})$ & $384 \mathrm{hr}(6,12 \mathrm{hr})$ & $240 \mathrm{hr}(12 \mathrm{hr})$ \\
\hline
\end{tabular}

MCGE forecasts that we have constructed using above three ensembles are listed in Table 2. As can be seen from Table 2, the alphabet indicates center name, namely J, N and C indicate JMA, NCEP, and CMC, respectively, and the number behind each alphabet indicates the number of each ensemble members. So, for example, J25N11C17 consists of 25 ensemble members of JMA, 11 members of NCEP, and 17 members of CMC, and the total number of members is 53. J9N8C8, which is constructed to compare with JMA25, contains JMA ensemble control run, 4 perturbation pairs of JMA, 4 perturbation pairs of NCEP, and 4 perturbation pairs of CMC. Initial time of MCGE forecasts is set to 12 UTC. So, the effect of Lagged Averaged Forecasts (LAF) method is naturally contained in MCGE forecasts by using CMC or NCEP members whose initial times are not 12 UTC.

\section{Methods}

\subsection{Deterministic verifications}

We investigate the skill of ensemble mean forecasts of MCGE using Root Mean Square Error (RMSE) and Anomaly Correlation (AC). RMSE is defined by the following equation:

$$
\mathrm{RMSE}=\sqrt{\frac{1}{N} \sum\left(x_{f}-x_{a}\right)^{2}},
$$

where $x_{f}$, and $x_{a}$ indicate the ensemble mean forecast and the analysis, respectively. In this study, $N$ is the number of grid points in the spatio-temporal domain, namely which indicates all grid points in the Northern Hemisphere $\left(20^{\circ} \mathrm{N}-90^{\circ} \mathrm{N}\right)$ in September 2005. Although calculating RMSE requires the analysis data, we regarded the JMA control run at initial time as the analysis data except for NCEP11 and CMC17. RMSE indicates a forecast error, and RMSE score of zero (0.0) demonstrates perfect skill.

$\mathrm{AC}$ is defined by the following equation:

$$
\mathrm{AC}=\frac{\sum\left(x_{f}-x_{c}\right)\left(x_{a}-x_{c}\right)}{\sqrt{\sum\left(x_{f}-x_{c}\right)^{2}} \sqrt{\sum\left(x_{a}-x_{c}\right)^{2}}},
$$

where $x_{f}, x_{a}$, and $x_{c}$ indicate ensemble mean forecast, analysis, and climate, respectively, and the summation is taken over the Northern Hemisphere. AC indicates a patterns correlation between forecast anomaly and analysis anomaly, so AC decreases with time. AC is 1.0 for the perfect forecast. Based on experience with the anomaly correlation, a score above 0.6 suggests that the forecast is sufficiently good, while a score below 0.6 signifies the forecast is not useful. In general, the time when AC first reaches 0.6 is called the limitation of predictability (hereafter referred to as LP). Calculating AC requires not only forecast and analysis data but also climate data. Although AC is sensitive to the choice of the climatological reference, we use JMA climate data.
Table 2. Configurations of single-center ensembles and MCGEs. Left column is abbreviated ensemble name.

\begin{tabular}{cccc}
\hline Name & $\begin{array}{c}\text { JMA mem. } \\
\text { (UTC) }\end{array}$ & $\begin{array}{c}\text { NCEP mem. } \\
\text { (UTC) }\end{array}$ & $\begin{array}{c}\text { CMC mem. } \\
\text { (UTC) }\end{array}$ \\
\hline JMA25 & $25(12)$ & - & - \\
NCEP11 & - & $11(12)$ & - \\
CMC17 & - & - & $17(00)$ \\
J9N8C8 & $9(12)$ & $8(12)$ & $8(00)$ \\
J25N11C17 & $25(12)$ & $11(12)$ & $17(00)$ \\
J25N44C17 & $25(12)$ & $44(18,00,06,12)$ & $17(00)$ \\
\hline
\end{tabular}

Therefore, it must be noted that our ACs for NCEP11 and $\mathrm{CMC} 17$ are not exact but approximate values, whereas our RMSEs for NCEP11 and CMC17 are accurate for reasons of not requiring climate data.

\subsection{Probabilistic verifications}

The most commonly used verification diagnostic for probabilistic forecasts is the Brier Score (BS), originally introduced by Brier (1950) and described in its modified standard form by Wilks (1995) as:

$$
\mathrm{BS}=\frac{1}{N} \sum_{i=1}^{N}\left(p_{i}-o_{i}\right)^{2} .
$$

BS is essentially the mean square error for probability forecasts of an event, where $p_{i}$ and $o_{i}$ are forecast and observed probabilities, respectively. In the verification of ensemble forecast, $p_{i}$ indicates how many ensemble members could predict the occurrence of the event. $o_{i}$ takes values of unity when the event occured and zero when it did not occur. $N$ is the number of grid points in the spatio-temporal domain, as in RMSE. BS is 0.0 for the perfect probabilistic forecast in which $p_{i}=O_{i}$ for all $i$. $\mathrm{BS}$ is sensitive to climatological frequency of the event. Brier Skill Score (BSS), defined by the following equation, is often used to measure the improvement of probabilistic forecast relative to climatological frequency:

$$
\mathrm{BSS}=\frac{\mathrm{BS}_{c l m}-\mathrm{BS}}{\mathrm{BS}_{c l m}}=1-\frac{\mathrm{BS}}{\mathrm{BS}_{c l m}},
$$

where $\mathrm{BS}_{c l m}$ is a BS for climate forecast. BSS becomes one (1.0) only when probabilistic forecast is perfect. Also BSS becomes zero (0.0) when probabilistic forecast is equivalent to climate forecast, indicating probabilistic forecast has no skill. Calculating BSS requires a threshold which is defined for an event. So, we configured 4 thresholds for Z500, whether anomaly is greater (less) than 1 or $2(-1$ or -2$)$ climatological standard deviation (hereafter referred to as SD), and 6 thresholds for T850, whether anomaly is greater (less) than 2,4 or $8(-2,-4$ or -8) K.

\section{Results}

\subsection{Comparison of the JMA, NCEP, and CMC ensemble forecasts}

First, we make a comparison among the skills of three ensemble mean forecasts, JMA25, NCEP11, and CMC17, using RMSE, AC, and BSS. When forecast skills of three centers are close to one another, the MCGE forecasts don't depend upon the skill of ensemble from single center.

RMSE was calculated for Z500 and T850 in the Northern Hemisphere using each analysis data. RMSEs for Z500 (T850) at $168 \mathrm{hr}$ of JMA25, NCEP11, and CMC17 are 55.6, 56.1, and $58.5 \mathrm{~m}$ (2.69, 2.75 and $2.86 \mathrm{~K})$, respectively. Also, AC was calculated for Z500 in the 


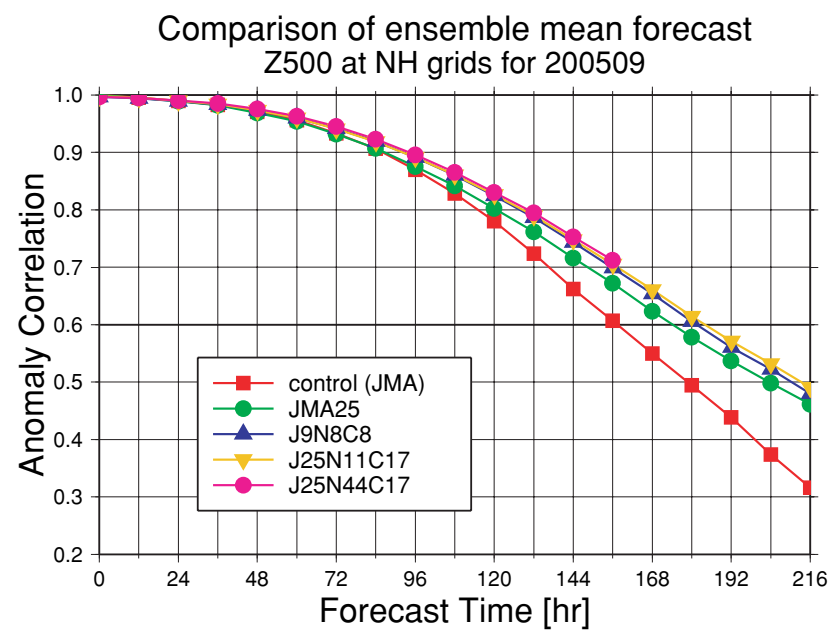

Fig. 1. Anomaly correlation skills for JMA control run and ensemble mean forecasts, JMA25, J9N8C8, $\mathrm{J} 25 \mathrm{~N} 11 \mathrm{C} 17$, and J25N44C17, Z500 in the Northern Hemisphere $\left(20^{\circ} \mathrm{N}-90^{\circ} \mathrm{N}\right)$ in September 2005 .

Northern Hemisphere using JMA climate and each analysis data. The monthly mean LP, when AC reaches 0.6 first, of JMA25, NCEP11, and CMC17 are $174 \mathrm{hr}, 180$ $\mathrm{hr}$, and $168 \mathrm{hr}$, respectively (not shown).

In the probabilistic verifications using BSS, we found that the skills of the three probabilistic forecasts are equivalent for many thresholds although the numbers of ensemble members are different from each other. However, in the case of the verification of climatologically rare events, such as BSS for the threshold whether T850 anomaly is less than $-8 \mathrm{~K}$, the skills of the three centers sometimes become quite different.

Looking overall, we can consider that the skills of three ensemble forecasts are almost equivalent. So, when we construct MCGE forecasts, the ensemble members are weighted equally.

\subsection{Effects of MCGE forecasts and increasing ensemble members in MCGE forecasts}

Figure 1 illustrates ACs of JMA control run, JMA25, J9N8C8, J25N11C17, and J25N44C17 for Z500. LP of J9N8C8 is $180 \mathrm{hr}$, which indicates MCGE is more skillful than JMA25 in the latter half of the forecast period. J25N11C17 seems to be slightly skillful than J9N8C8. It is interesting that in spite of LPs of JMA25, NCEP11, and $\mathrm{CMC} 17$ are $174 \mathrm{hr}, 180 \mathrm{hr}$, and $168 \mathrm{hr}$, respectively, that of J25N11C17 exceeds $180 \mathrm{hr}$, which is maximum within three single-center ensemble forecasts. Also it is expected from Fig. 1 that J25N44C17 is slightly skillful than J25N11C17 in the latter half of the forecast period, although J25N44C17 cannot be constructed over $156 \mathrm{hr}$ lead time owing to the forecast intervals. However, the difference among MCGE forecasts, J9N8C8, J25N11C17, and J25N44C17, namely which indicates the effects of increasing ensemble members, is smaller than that between JMA25 and J9N8C8, which indicates the effects of multi-model. Similar results are obtained with respect to RMSE for Z500 and T850 (not shown). RMSEs for Z500 (T850) at $168 \mathrm{hr}$ of J9N8C8 and J25N11C17 are 53.5 and $53.1 \mathrm{~m}(2.51$ and $2.46 \mathrm{~K})$, respectively. Also, RMSEs for Z500 and T850 at $156 \mathrm{hr}$ of J25N44C17, which cannot be constructed over 156 hr lead time, are the smallest in MCGEs.

Figure 2 illustrates BSSs of JMA25, J9N8C8, J25N11C17, and J25N44C17 for the threshold whether
Brier Skill Score 200509 Z500 anomaly greater than $1 \mathrm{SD}$ at $\mathrm{NH}$ grid

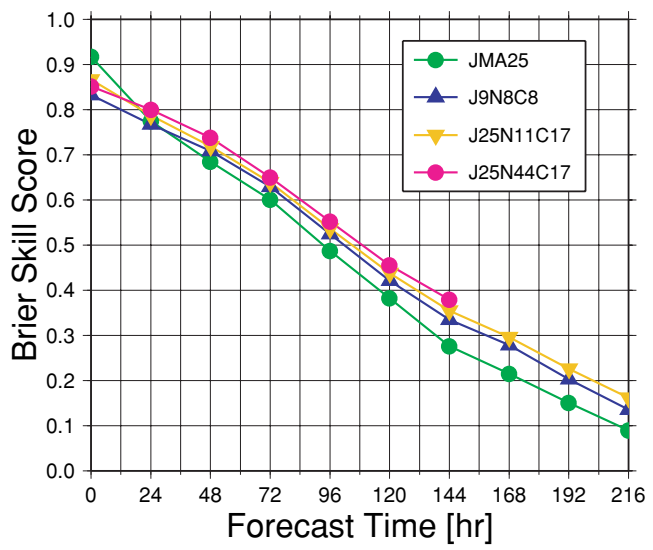

Fig. 2. BSS of probabilistic predictions, JMA25, J9N8C8, J25N11C17, and J25N44C17, for Z500 in the Northern Hemisphere $\left(20^{\circ} \mathrm{N}-90^{\circ} \mathrm{N}\right)$ in September 2005. The threshold is whether the anomaly is greater than $1 \mathrm{SD}$.

Z500 anomaly is greater than 1 SD. BSSs of MCGEs after $+24 \mathrm{~h}$ forecast time is superior to that of JMA25, whereas that up to $+24 \mathrm{~h}$ forecast time are inferior to that of JMA25. J25N11C17 is more skillful than J9N8C8, and also J25N44C17 is more skillful than J25N11C17 in the latter half of the forecast period. The same result is obtained with respect to the threshold whether anomaly is greater than $2 \mathrm{SD}$ (not shown), except for the similar values of JMA25 and J9N8C8 in the middle of the forecast (48 hr-120 hr). However, for -1 or -2 SD thresholds there is no apparent effect of increasing MCGE members, although all MCGEs are superior to JMA25.

Figure 3 illustrates BSSs of JMA25, J9N8C8, J25N11C17, and J25N44C17 for the threshold whether T850 anomaly is less than $-4 \mathrm{~K}$. MCGEs are remarkably superior to JMA25 after +24 h forecast time. However, BSS of J25N11C17 is almost equal to that of J9N8C8, and J25N44C17 is slightly superior to these. The same result is obtained with respect to the thresholds whether anomaly is less than -2 or $-8 \mathrm{~K}$ (not shown), except that J25N11C17 is superior to J9N8C8 for $-8 \mathrm{~K}$ thresholds. With respect to T850, 2, 4 or $8 \mathrm{~K}$ thresholds, MCGEs are not always superior to JMA25 even in the latter half of the forecast period (not shown). J9N8C8 is comparable to or inferior to JMA25, whereas J25N11C17 and J25N44C17 are mostly superior to JMA25 except for disadvantage of J25N44C17 compared to JMA25 with respect to $8 \mathrm{~K}$ threshold. Also, $\mathrm{J} 25 \mathrm{~N} 44 \mathrm{C} 17$ is not superior to J25N11C17 for 4 or $8 \mathrm{~K}$ thresholds, whereas J25N44C17 is slightly superior to J25N11C17 for $2 \mathrm{~K}$ threshold.

\section{Conclusions}

We investigate the impact of MCGE forecasts, consisting of three operational ensemble forecasts by JMA, $\mathrm{NCEP}$, and $\mathrm{CMC}$. In this paper, we show the verification results of MCGEs in comparison with that of JMA ensemble forecast using RMSE, AC, and BSS for Z500 and T850 in the Northern Hemisphere $\left(20^{\circ} \mathrm{N}-90^{\circ} \mathrm{N}\right)$ in September 2005.

First, we made a comparison among the skills of ensemble mean forecasts from the three centers. According to the deterministic and probabilistic verifi- 
Brier Skill Score 200509 T850 anomaly less than $-4 \mathrm{~K}$ at $\mathrm{NH}$ grid

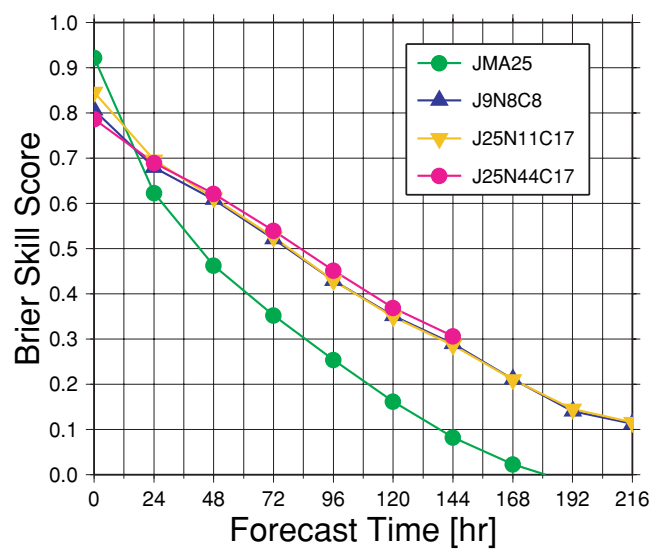

Fig. 3. Same as Fig. 2 but for T850. The threshold is whether anomaly is less than $-4 \mathrm{~K}$.

cations using RMSE, AC, and BSS, we found that the skills of three operational ensemble forecasts are almost equivalent to each other. So, when we construct MCGEs, the ensemble members were weighted equally.

Next, we compared a MCGE with JMA ensemble, whose ensemble size is 25 . The deterministic verifications using RMSE and AC indicate that MCGE is more skillful than single-center ensemble. Also, the probabilistic verification using BSS indicates that MCGE is more skillful than single-center ensemble although BSSs of MCGE for T850 threshold, whether anomaly is greater than 2, 4 or $8 \mathrm{~K}$, are almost equivalent or inferior to that of JMA ensemble.

Furthermore, we investigated the effects of increasing ensemble members in MCGEs. The deterministic verifications using RMSE and AC indicate that the more ensemble size increases, the more skillful MCGEs are. However, these effects are not so much large as the previous effects of MCGE.

In probabilistic verification for Z500, 1 or $2 \mathrm{SD}$ and T850, $-2,-4$ or $-8 \mathrm{~K}$ thresholds, there are effects of increasing ensemble members. However, for Z500, -1 or -2 SD thresholds MCGEs have same forecast skill. Also, the effects are not always detectable by increasing members for 2, 4 or $8 \mathrm{~K}$ thresholds even in the latter half of the forecast period. If anything, MCGE with the largest ensemble size is sometimes less skillful than the other MCGEs for climatologically rare events. Although there are some differences by thresholds, the effects of increasing members are not so much large as the effects of MCGE.

\section{Discussions}

As described in the previous section, it is found that MCGE forecasts are more skillful than single-center ensemble forecast without weight among ensemble members and bias corrections. Although the effects of increasing members in MCGEs are not so much large as the effects of MCGE, this might be a result from containing ensemble members whose initial time is not 12 UTC, such as NCEP ensemble forecasts (18, 00, 06 UTC) and $\mathrm{CMC}$ ensemble forecast (00 UTC). If we can obtain further ensemble forecast data, whose initial time is 12 UTC, under combined efforts of other NWP centers or further developments of computer resource, one will be able to construct more skillful MCGEs and perform further significant analyses on MCGEs.

Also, considering weight or bias corrections may lead to further improvement of MCGEs, specifically in probabilistic forecasts. Especially, when forecast skill of certain center is extremely worse than that of the other two centers, we can construct MCGEs using two centers ignoring the third one.

Although we found the superiority of MCGEs for September 2005, it is interesting to investigate the seasonal variation in the impacts of MCGE. In addition, in order to answer the question why MCGEs can beat a single-center ensemble, it may be necessary to analyze not monthly mean forecast skills but individual forecast events.

Further, it is interesting to investigate the forecast skills of MCGEs, consisting of many more ensemble members obtained from further NWP centers. Possibly, the forecast skills of MCGEs may peak out at a particular number of members. If anywhere, the studies on MCGEs cannot be done without combined efforts of other NWP centers.

\section{Acknowledgments}

This work could not have been done without the operational ensemble data of NCEP and CMC. We are especially thankful to NCEP and CMC. A part of the research support comes from IARC/UAF.

\section{References}

Brier, G. W., 1950: Verification of forecasts expressed in terms of probability. Mon. Wea. Rev., 78, 1-3.

Buizza, R., D. S. Richardson, and T. N. Palmer, 2003: Benefits of increased resolution in the ECMWF ensemble system and comparison with poor-man's ensembles. Quart. J. Roy. Meteor. Soc., 129, 1269-1288.

Houtekamer, P. L., L. Lefaivre, J. Derome, H. Ritchie, and H. L. Mitchell, 1996: A system simulation approach to ensemble prediction. Mon. Wea. Rev., 124, 1225-1242.

JMA, 2002: Outline of the operational numerical weather prediction at the Japan Meteorological Agency. Japan Meteorological Agency, 158 pp.

Kalnay, E., 2003: Atmospheric modeling, data assimilation and predictability. Cambridge University Press, Cambridge, $341 \mathrm{pp}$.

Krishnamurti, T. N., C. M. Kishtawal, T. E. LaRow, D. R. Bachiochi, Z. Zhang, C. E. Williford, S. Gadgil, and S. Surendran, 1999: Improved weather and seasonal climate forecasts from multimodel superensemble. Science, 285, 1548-1550.

Molteni, F., R. Buizza, T. N. Palmer, and T. Petroliagis, 1996: The ECMWF ensemble prediction system: Methodology and validation. Quart. J. Roy. Meteor. Soc., 122, 73-119.

Mylne, K. R., R. E. Evans, and R. T. Clark, 2002: Multi-model multi-analysis ensembles in quasi-operational mediumrange forecasting. Quart. J. Roy. Meteor. Soc., 128, 361384.

Richardson, D. S., 2001: Ensembles using multiple models and analyses. Quart. J. Roy. Meteor. Soc., 127, 1847-1864.

Toth, Z., and E. Kalnay, 1997: Ensemble forecasting at NCEP and the breeding method. Mon. Wea. Rev., 125, 32973319.

Wilks, D. S., 1995: Statistical methods in the atmospheric sciences. An introduction. International Geophysics Series Vol. 59, Academic Press, 467 pp.

Ziehmann, C., 2001: Comparison of a single-model EPS with a multi-model ensemble consisting of a few operational model. Tellus, 52A, 280-299.

Manuscript received 19 December 2005, accepted 7 February 2006 SOLA: http://www.jstage.jst.go.jp/browse/sola/ 